\title{
Erratum to: Presidential Healthcare Reform Rhetoric: Continuity, Change \& Contested Values from Truman to Obama
}

\author{
Noam Schimmel
}

(C) The Author(s) 2016

Noam Schimmel, Presidential Healthcare Reform Rhetoric, Rhetoric, Politics and Society, DOI 10.1007/978-3-319-32960-4

DOI 10.1007/978-3-319-32960-4

In the previous version, originally published in September 2016, crossreferences that should have been revised following an earlier decision to remove the appendix were left unchanged. In the present version they now refer the reader to the relevant online sources. During the post-publication revision process the opportunity was also taken to make other minor corrections and review and update the URL links. The previous publication does not reflect the intended final product.

The updated online version of the original book can be found under DOI 10.1007/978-3-319-32960-4 